\title{
Assembly and Dynamic Analysis of Square Colloidal Crystals via Templated Capillary Assembly
}

\author{
Cicely Shillingford ${ }^{1}$, Veronica Grebe ${ }^{1}$, Angus McMullen², Jasna Brujic ${ }^{2, *}$, and Marcus Weck ${ }^{1, *}$ \\ ${ }^{1}$ Molecular Design Institute and Department of Chemistry, New York University, New York, NY 10003, USA \\ ${ }^{2}$ Center for Soft Matter Research and Department of Physics, New York University, New York, NY 10003, USA
}

\section{Supplementary Information}

\section{Materials}

Silicon and glass wafers were purchased from University Wafer and chromium photomasks were ordered from DigiDat. Gold pellets, chromium pellets, and tungsten baskets used for metal evaporation were acquired from Ted Pella, Inc. SU-8 photoresists, and the corresponding developers were all purchased from MicroChem. DNA containing the sequences 5'-DBCO- $\mathrm{T}_{20}-\mathrm{Cy}_{3}-\mathrm{T}_{80}-\mathrm{TGCGGT}-3$ ' and 5'-ThioMC6-D- $\mathrm{T}_{20}-\mathrm{Cy}_{3}-\mathrm{T}_{80}-\mathrm{ACCGCA}-3^{\prime}$ was purchased from Integrated DNA Technologies (IDT), Inc. 3-(trimethoxysilyl)propyl methacrylate (TPM, 98\%) and ammonium hydroxide (35\%) were purchased from Sigma-Aldrich ${ }^{\circledast}$ and used without modification. All other reagents and solvents were obtained from Sigma-Aldrich ${ }^{\circledast}$ and all were used without further modification. Deionized water was obtained from a Millipore Filtration System (Milli-Q gradient A1o). Optical microscopy, scanning electron microscopy (SEM), and confocal microscopy were procured from a Nikon TE3oo microscope, a Merlin (Carl Zeiss) field-emission SEM, and a Leica SP $_{5}$ confocal fluorescence microscope respectively. Contact angle measurements were performed on an Attension ${ }^{\circledast}$ Theta optical tensiometer.

\section{Supplementary Methods}

\section{Grafting of DNA onto particles}

Synthesis of DNA modified colloids was enabled by the use of 3-chloro-2-hydroxypropyl methacrylate (TPM-Cl) to create a surface decorated with functional handles. TPM-Cl $(80 \mu \mathrm{L})$ was added to the liquid emulsion after six hours and stirred for 30 minutes before adding $5 \mathrm{~mL}$ of $5 \mathrm{wt} \%$ F108 (poly(ethylene glycol)-block-poly(propylene glycol)block-poly(ethylene glycol)). The particles were polymerized and washed $5 \mathrm{x}$ with water to remove residual surfactant, diluted to $0.5 \mathrm{wt} \%$, and centrifuged into a pellet in an Eppendorf tube. Sodium azide (5 mg) and potassium iodide $(5 \mathrm{mg}$ ) were mixed with $1.5 \mathrm{~mL}$ of $1 \mathrm{wt} \%$ F127 in water; this solution is poured over the pellet, sonicated, and placed on a thermal shaker at $70{ }^{\circ} \mathrm{C}$ overnight. The azide-functionalized particles were washed $4 \mathrm{x}$ with fresh 1 wt $\%$ F127.

DNA was grafted onto the particles using strain-promoted azide-alkyne coupling (SPAAC) of a diarylcyclooctyne (DBCO) functionalized DNA strand with an internal Cyanine-3 (Су3) fluorescent dye and a sticky end with the sequence TGC GGT. $400 \mu \mathrm{L}$ of azide-functionalized particles were washed into $10 \mathrm{mM}$ phosphate buffered saline (PBS, $\mathrm{pH}=7.4,500 \mathrm{mM} \mathrm{NaCl}$ ) containing $0.1 \%$ Triton $\mathrm{X}-100$ and mixed with $10 \mu \mathrm{L}$ of a $1 \mathrm{nM}$ stock solution. The samples were shaken at $40^{\circ} \mathrm{C}$ overnight, washed with fresh water and imaged via confocal microscopy. The Cy3 dye has an emission maximum of $564 \mathrm{~nm}$ (Figure $\mathrm{S}_{3}$ ).

\section{Gold template fabrication with DNA grafting}

Glass substrates were cleaned with acid piranha $\left(3: 1 \mathrm{H}_{2} \mathrm{SO}_{4}: \mathrm{H}_{2} \mathrm{O}_{2}\right)$ for $1 \mathrm{~h}$ at RT to remove organic impurities. The wafers were coated with $5 \mathrm{~nm}$ of chromium and 20-50 nm of gold in a thermal evaporator at a rate of $0.05-0.2 \mathrm{~nm} / \mathrm{s}$. The gold substrates were rinsed with acetone, dried with $\mathrm{N}_{2}$, and cleaned with air plasma for two minutes. Fabrication of patterns via photolithography was carried out as described above to give templates with exposed gold at the bottom of each well. ThioMC6-D (a dithiol modifier) DNA ( $5 \mu \mathrm{L}, 1 \mathrm{nM}$ stock solution) was reduced by 
shaking of in $300 \mathrm{x}$ excess tris[2-carboxyethyl] phosphine (TCEP) and PBS (100 $\mu \mathrm{L}, 10 \mathrm{mM}$ ) for four hours at room temperature. The reduced DNA was immediately injected into the gold functionalized flow cells, sealed, and shaken overnight. The flow cells were rinsed with water to remove unbound DNA and stored at $4{ }^{\circ} \mathrm{C}$ in PBS (10 mM) until ready to use. 


\section{Supplementary Tables}

Table S1. Calculated volume fractions associated with particle concentrations using $\rho_{\mathrm{TPM}}=1 \cdot 314(4) \mathrm{g} \mathrm{cm}^{-3} .^{1,2}$

\begin{tabular}{lr}
\hline \multicolumn{1}{c}{ Weight $\%$} & \multicolumn{1}{c}{$\phi$} \\
0.50 & 0.005 \\
1.00 & 0.008 \\
1.50 & 0.011 \\
1.75 & 0.013 \\
2.00 & 0.015 \\
2.25 & 0.017 \\
2.50 & 0.019 \\
3.00 & 0.023 \\
\hline
\end{tabular}




\section{Supplementary Figures}

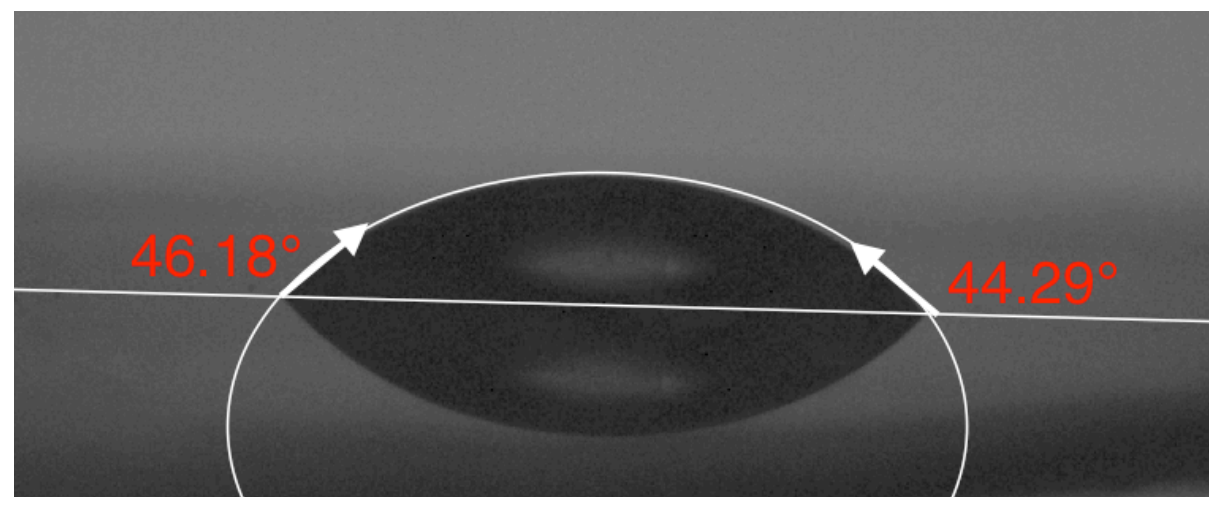

Figure S1. Representative contact angle of a $5 \mu \mathrm{L}$ droplet of $1.0 \mathrm{wt} \%$ aqueous F1o8 on an SU-8 patterned glass wafer substrate. The average static contact angle recorded was $45(2)^{\circ}$. Imaging and analysis was completed on an Attension ${ }^{\circledR}$ Theta Optical Tensiometer.

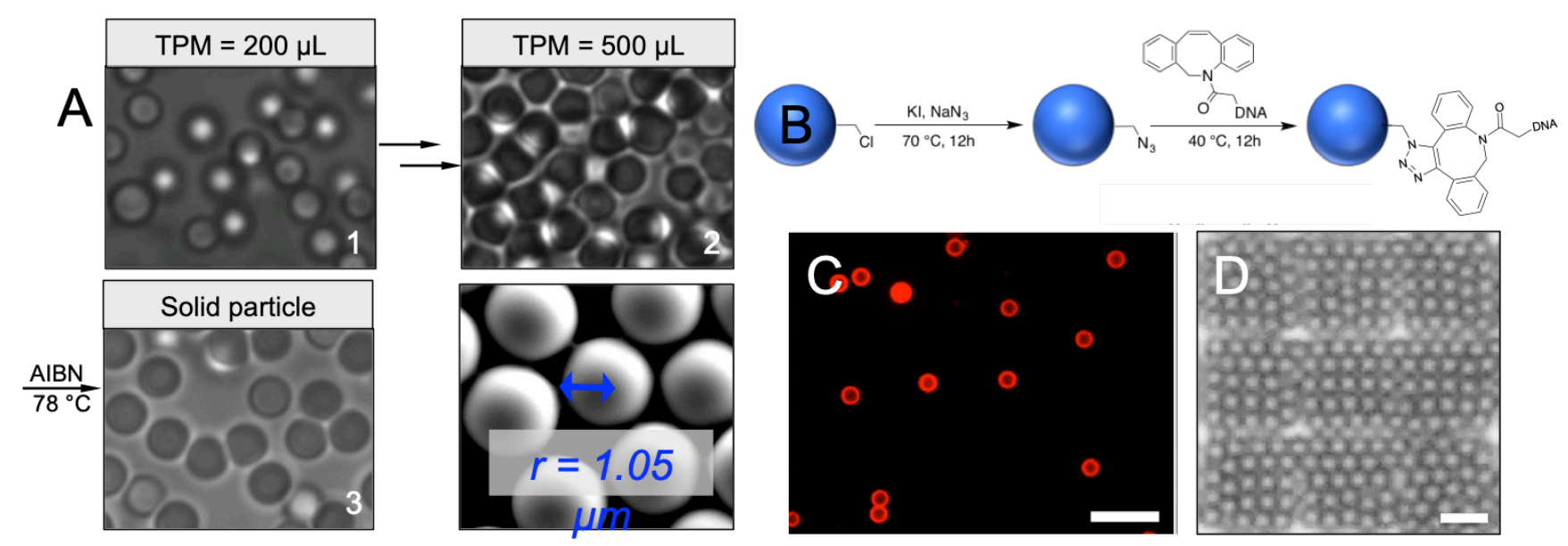

Figure S2. (A) TPM particle synthesis via a condensation and emulsion droplet swelling followed by radical polymerization. Particles produced have radii of $1 \mu \mathrm{m}$. (B) Scheme showing the functionalization of TPM-Cl particles first with an azide moiety followed by SPAAC to a DBCO modified DNA molecule. The DNA contains the fluorescent dye Cy5 (red). (C) Successful DNA grafting was confirmed by confocal microscopy. (D) Capillary assembly result using DNA coated particles with complementary DNA modified templates; : $\phi_{\mathrm{p}}, \mathrm{zn}=4=0.29(0.02)$. Scale bar $=6 \mu \mathrm{m}$. 


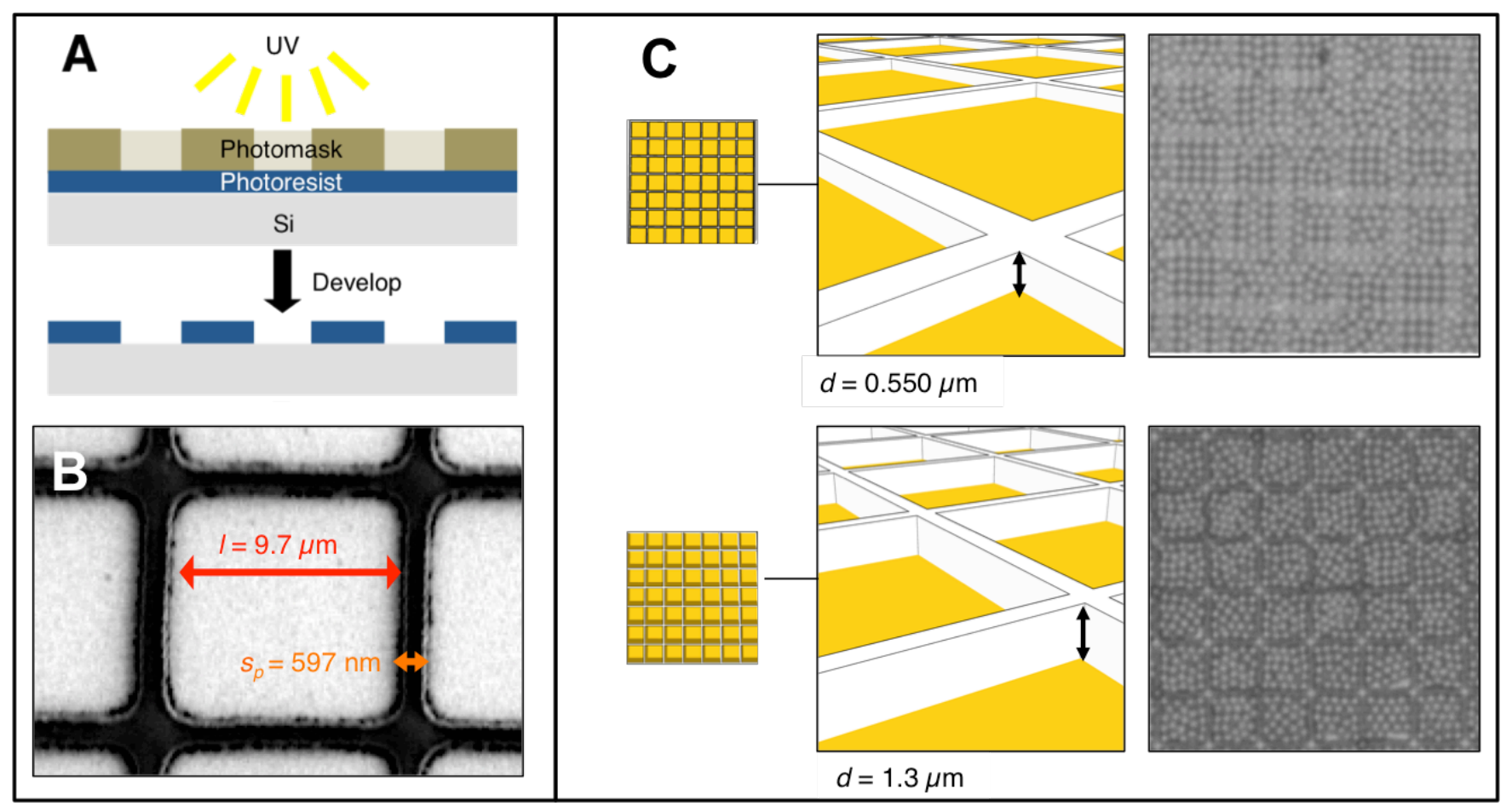

Figure S3. (A) General scheme showing fabrication of glass patterned templates using photolithography. (B) Square wells made from negative photoresist SU-8 2000.5 on silicon; $l=w=9.7(0.3) \mu \mathrm{m}, s_{p}=597(10) \mathrm{nm}$. (C) Using a deeper template for crystallization in which $\mathrm{d} \geq \mathrm{r}$ results in discrete boxes containing either HCP or amorphously packed colloids. Colloids are not able to bridge the boundary to form a continuous crystal. 


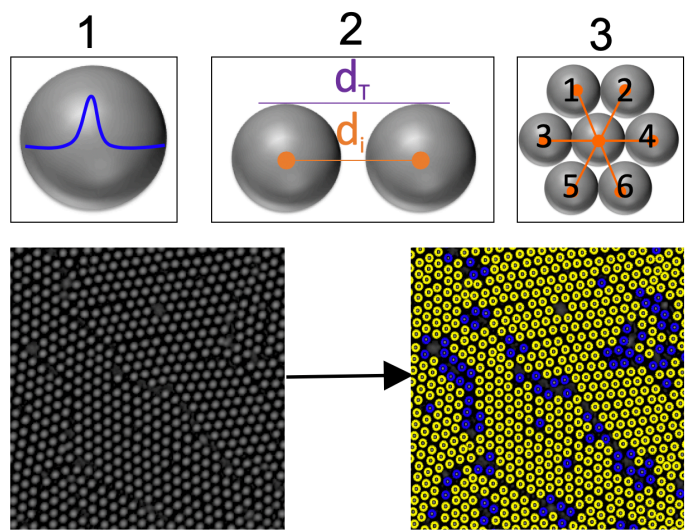

Figure S4. Quantifying particle packing using MatLab. (1) A Gaussian filter is applied to all particles, and the peak of the Gaussian for each particle is used to locate the individual particle centers. (2) The Euclidean distance $\left(\mathrm{d}_{\mathrm{i}}\right)$ between each particles' centers is calculated. A distance threshold $\left(\mathrm{d}_{\mathrm{T}}=20\right)$ is determined to fit the following criteria to ensure only adjacent particles will be classified as neighbors: $d_{T} \geq d_{i}$ for all neighboring particles, and $d_{T}$ is not greater than or equal to the Euclidean distance between any two non-neighboring particles' centers. If $\mathrm{d}_{\mathrm{T}} \geq \mathrm{d}_{\mathrm{i}}$ for any two particles, the particles are classified as neighbors and are considered coordinated. (3) The summation of the amount of particles that are classified as neighbors is defined as the coordination number for the individual particles. A matrix defining particle coordination is generated by determining the amount of particles that neighbor any given particle. A color-coded image is generated over the original gray scale image that maps the coordination number to each individual particle. Yellow particles have $Z_{n}=5-6$, blue particles have $Z_{n}=3-4$.

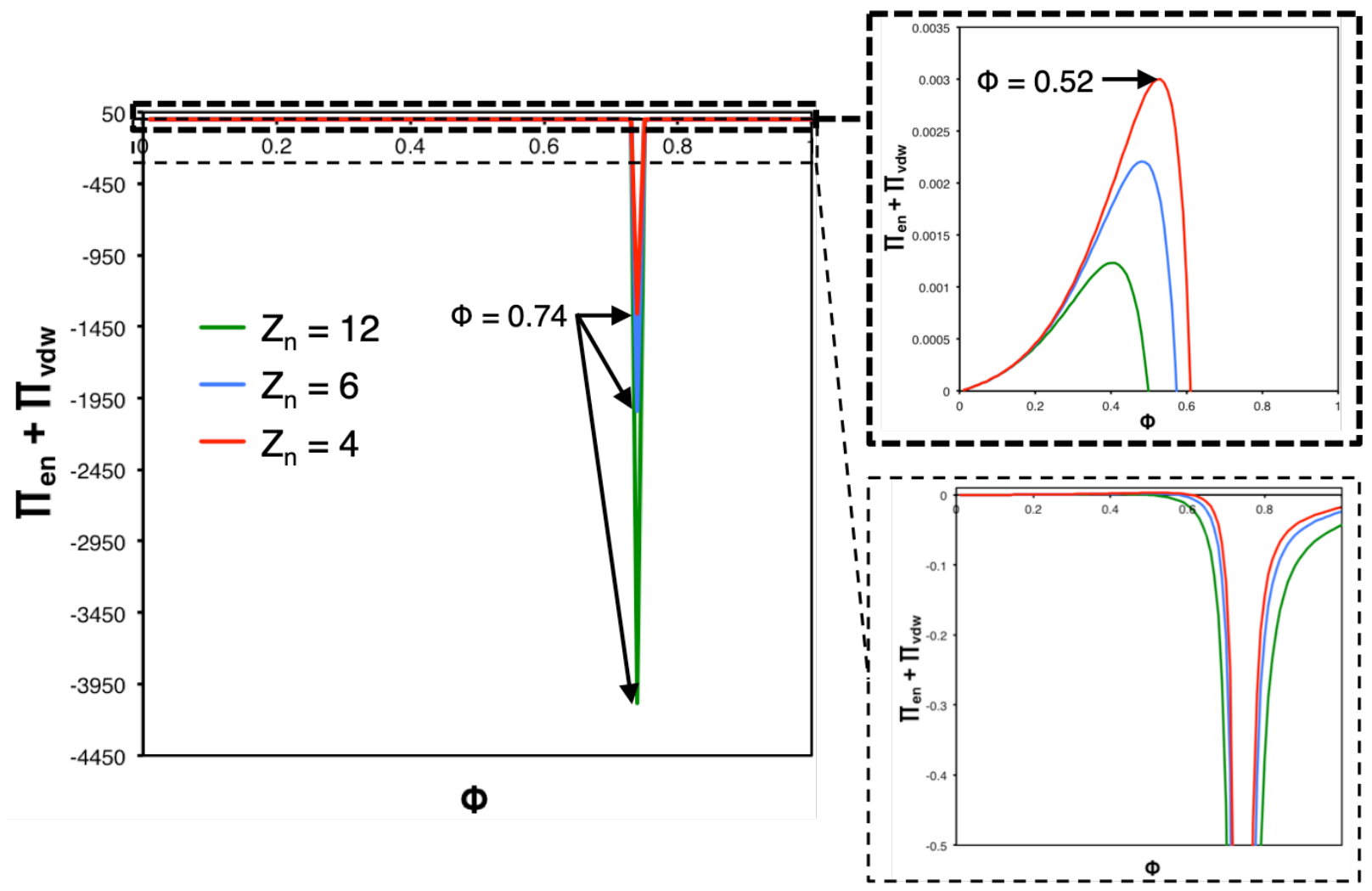

Figure S5. Osmotic pressure versus volume fraction plots. The entropic and Van der Waals osmotic pressure contributions were calculated as described by Wolff and coworkers. ${ }^{3}$ Plotting the sum of these two terms against the volume fraction for different coordination numbers $\left(Z_{n}\right)$ revealed that $\prod_{e n}+\prod_{v d w}$ is minimal at $\phi=0.74$ for all values of $Z_{n}$, which is the packing efficiency for an FCC crystal. For $\mathrm{Zn}=4, \prod_{\mathrm{en}}+\prod_{\mathrm{vdw}}$ is maximal at $\phi=0.52$, which corresponds to the packing efficiency of a simple cubic lattice. 


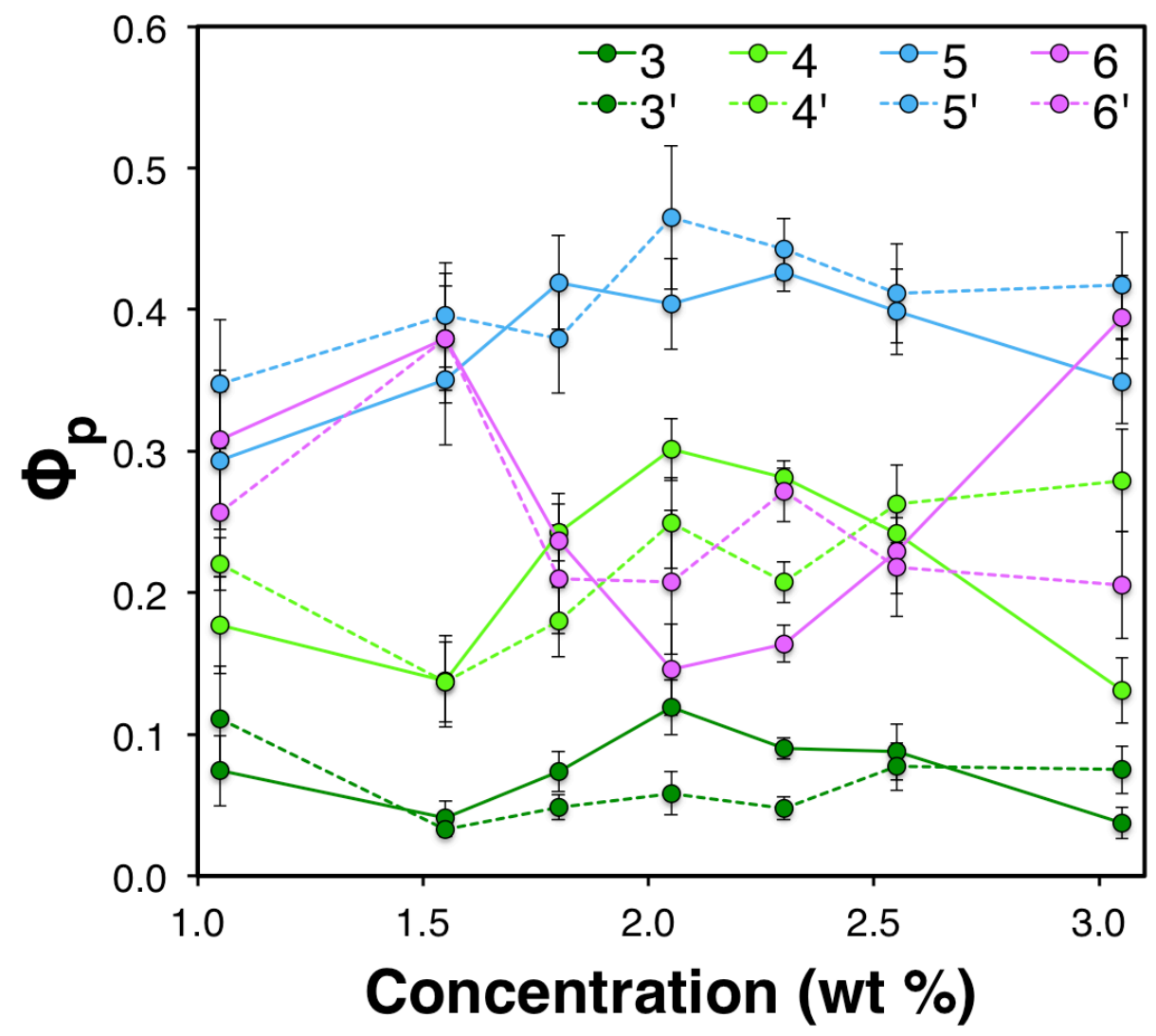

Figure S6. Plots of $\phi_{p}$ versus concentration for $Z_{n}=3^{-6}$ for substrate depths of $400 \mathrm{~nm}$ and $550 \mathrm{~nm}$. Reducing the template depth reduces the variance of particles occupying different coordination environments.

\section{Supplementary Video Captions}

Video S1. Representative templated capillary assembly process using conditions described in Table $\mathrm{S}_{1}$ with $\mathrm{H}_{2} \mathrm{O}$ as the solvent. The video was recorded at 10 frames per second (fps) and is displayed at $5 \mathrm{x}$ speed. The red box indicates an area in which restructuring occurs, its dimensions are $10 \mu \mathrm{m} \times 10 \mu \mathrm{m}$.

Video S2. Coordination number mapping of capillary assembly demonstrating that particles undergo phase transitions throughout solvent evaporation. This video corresponds to the data in Figure ${ }_{4} \mathrm{C}$. In this instance so as to more clearly delineate HCP particles from the masses, yellow particles represent $Z_{n} \geq 6$, blue particles represent $Z_{n}=1-5$.

Video $S_{3}$. Representative templated capillary assembly process using conditions described in Table $\mathrm{S}_{1}$ with $\mathrm{D}_{2} \mathrm{O}$ as the solvent. The video was recorded at 10 frames per second (fps) and is displayed at $5 \mathrm{x}$ speed.

Video S4. Representative templated capillary assembly process using conditions described in Table $\mathrm{S}_{1}$ with $\mathrm{H}_{2} \mathrm{O}$ as the solvent except for that the template depth equals $1.3 \mu \mathrm{m}$. The video was recorded at 10 frames per second (fps) and is displayed at 5x speed. 


\section{Supplementary Discussion}

\section{Particle coordination mapping on MatLab}

A custom MATLAB script was used to locate particles within the image and assign coordination numbers to each individual particle. Once the greyscale .tif image of the particles is loaded in, a Gaussian filter is applied. A bandpass defining a pixel size larger than that of the particle is used to find the peak of the Gaussians, which effectively locates each particle based on contrast intensity. After first defining the distance between each particle, a coordination matrix is then generated by determining how many particles are connected to surrounding particles within the defined interparticle distance. A colormap is then applied over the greyscale image to display each particle with its determined coordination, as determined by color. Data gathered via videos (10 fps) were separated into individual frames. Each individual frame was loaded into MATLAB, and the script was looped to analyze each frame.

\section{Effect of template resolution}

We confirmed the significance of template geometry by investigating the effects of modified shape and resolution. The particle and surfactant concentration as well as trap depth were fixed at $2.0 \mathrm{wt} \%$, $1.0 \mathrm{wt} \%$, and $550 \mathrm{~nm}$, respectively. Substrates with no template (i.e. a flat surface) produced complete HCP monolayers as expected. Employing square templates with large spacing $\left(s_{p}=4 \mu \mathrm{m}\right)$ delivered a mostly amorphous crystal, as did templates in which the boundary integrity was reduced by underexposing the photoresist. Adding curvature and breakage to the pattern precluded the formation of square nuclei; these results are shown in Figure S7.

\section{Osmotic pressure calculation}

The entropic and van der Waals osmotic pressure terms were calculated based on a method described by Wolf and coworkers. ${ }^{3}$ 


\section{References}

1. van der Wel, C.; Bhan, R. K.; Verweij, R. W.; Frijters, H. C.; Gong, Z.; Hollingsworth, A. D.; Sacanna, S.; Kraft, D. J. Preparation of colloidal organosilica spheres through spontaneous emulsification. Langmuir 2017, 33, 8174-8189.

2. Azmi, W. H.; Sharma, K. V.; Sarma, P. K.; Mamat, R.; Anuar, S.; Rao, V. D. Experimental determination of turbulent forced convection heat transfer and friction factor with $\mathrm{SiO}_{2}$ nanofluid. Exp. Therm. Fluid Sci. 2013, 51, 103-111.

3. Ni, S.; Leeman, J.; Wolf, H.; Isa, L. Insights into mechanisms of capillary assembly. Faraday Discuss. 2015, 181, 225242.

4. Malaquin, L.; Kraus, T.; Schmid, H.; Delamarche, E.; Wolf, H. Controlled particle placement through convective and capillary asembly. Langmuir 2007, 23, 11513-11521.

5. Flauraud, V.; Mastrangeli, M.; Bernasconi, G. D.; Butet, J.; Alexander, D. T. L.; Shahrabi, E.; Martin, O. J. F.; Brugger, J. Nanoscale topographical control of capillary assembly of nanoparticles. Nature Nanotechnology 2017, $12,73-81$.

6. Kraus, T.; Malaquin, L.; Schmid, H.; Riess, W.; Spencer, N. D.; Wolf, H. Nanoparticle printing with single-particle resolution. Nature Nanotechnology 2007, 2, 570-576. 